\title{
An Empirical Analysis of the Impact of Local Officials' Characteristics on Environmental Governance Performance
}

\author{
Feng Zhu ${ }^{1}$ \\ ${ }^{1}$ School of Economics and Management, East China Jiaotong University, Nanchang, Jiangxi 330013, China
}

\begin{abstract}
This paper uses China's 2010-2018 city-level panel data and the annual average PM2.5 concentration data processed by ArcGIS software and uses the LASSO regression model to empirically analyze the impact of local officials' characteristics environmental governance performance. The results show that younger officials, municipal party committee secretaries who graduated from ordinary colleges and universities, municipal party committee secretaries who have been vacated, and general mayors are more conducive to environmental governance; those who have worked in state-owned enterprises, are older, have studied The secretary of the municipal party committee and a mayor who is promoted from the grassroots in economics and management, the secretary of the municipal party committee with a bachelor's degree, the mayor who has a graduate degree, the mayor who has committed corruption and discipline, and the mayors who graduated from the party school are not conducive to the jurisdiction Environmental governance. The research results of this article help to understand the role of individual differences in local officials in environmental governance, and can also provide reference suggestions for cadres and personnel reform.
\end{abstract}

\section{Introduction}

Environmental governance is closely related to government behaviour, and government behaviour is influenced by officials. Although China's environmental problems have been greatly improved in recent years, they still need to be paid attention to. At present, the proxy variables used to measure the performance of local environmental governance in economic research are not uniform [1-3]. The more serious one is air pollution. The haze problem is the most prominent. However, PM2.5 data is rarely used as a proxy variable for environmental governance performance in existing studies. At present, some scholars have studied the impact of local officials' characteristics on environmental governance [4,5]. However, existing studies have selected many explanatory variables involving individual characteristics of officials, which easily leads to the emergence of multicollinearity, which leads to parameters. The economic meaning of the estimator is unreasonable, and the significance test of the variable loses its meaning and other serious consequences.

Aiming at the deficiencies of existing research, this paper uses PM2.5 data as a proxy variable for environmental governance performance. Because PM2.5 data is challenging to obtain, it is rarely used in economic research. This article uses the latest global atmospheric PM2.5 annual average concentration raster dataset released by the Canadian Dalhousie University Atmospheric Composition Analysis Group and uses ArcGIS software to process the annual average PM2.5 concentration. This data is more objective and covers China's Most prefecture-level cities. Because many variables added to the regression model can easily lead to multicollinearity, this paper adopts the LASSO regression model that can overcome multicollinearity to do empirical analysis, to screen out officials' characteristics will have a significant impact on the performance of urban environmental governance.

The purpose of this article is to explore which individual characteristics of officials will affect the performance of environmental governance, whether the impact is positive or negative, and how large is the impact. This article's research results help to understand the role of individual differences in local officials in environmental governance and can also provide reference suggestions for cadres and personnel reforms.

\section{Research Design}

\subsection{Data Sources}

This article uses panel data from 285 prefecture-level cities in China from 2010 to 2018 and matches the municipal party committee secretary and mayor's official information in detail. The original data of each city's annual average PM2.5 comes from the atmospheric composition analysis group of Dalhousie University. Compared with the data released by Columbia University, the satellites used are all NASA satellites, and the data is comprehensively estimated using data from ground monitoring stations. The data has not been subjected to three-year sliding processing, and more ground monitoring data is used to assist satellite data, which is 
relatively more accurate. The original data are processed by ArcGIS software to get the annual average PM2.5 data of prefecture-level cities used in this article. The personal information of the secretary of the municipal party committee and the mayor of a prefecture-level city, such as official age, replacement, resume, education experience, etc., is manually searched and sorted based on online resources such as Xinhuanet and Baidu Baike. Data such as per capita GDP, secondary industry, and urban FDI share are derived from the "China City Statistical Yearbook" of each year.

\subsection{Variable description}

\subsection{1 explained variable}

This paper uses the annual average PM2.5 concentration (pm25) of 285 prefecture-level cities in China from 2010 to 2018 as the explained variable.

\subsection{2 core explanatory variables}

Based on the research done by previous scholars, three types of explanatory variables are selected in this article (where the variable prefix a represents the secretary of the municipal party committee, and the variable prefix $b$ represents the mayor): (1) Individual characteristics of officials: including officials The gender ( $a x b, b x b)$, age (anl, bnl), corruption, violation of discipline, etc. (alm, blm). (2) The educational background of the officials: whether the final degree is a bachelor degree (abk, bbk), whether their final degree is a postgraduate degree (ayjs, byjs), whether they graduated from ordinary colleges and universities (apg, bpg), whether they graduated from party schools at all levels $(\mathrm{adx}, \mathrm{bdx})$, whether they have studied economics and management majors (ajg, bjg). (3) The official' $s$ background: including whether they had only work experience at the municipal level or below before serving as secretary of the municipal party committee or mayor. This variable is referred to as grassroots promotion (ajctb, bjctb); Whether they have only work experience in an administrative unit above the municipal level before serving as the secretary of the municipal party committee or mayor(axjkp, bxjkp), Whether they have worked in a unit at or above the city level before being promoted as secretary of the municipal party committee or mayor, or whether they have had work experience in a unit at or below the municipal level before being airborne as secretary of the municipal party committee or mayor. This variable is referred to as comprehensive appointment (azhrz , bzhrz), whether they have ever worked in a stateowned enterprise (agq, bgq), whether they have served as a principal (axz, bxz), above all dummy variables take a value of 1 to indicate yes, a value of 0 to indicate no, a value of 1 for the gender variable to indicate a male, and a value of 0 to indicate a female.

\subsection{3 control variables}

To make the estimation results of this article more reliable, this article draws on the practice of Cai Fang et al. [6], adding the following control variables (1) the ratio of secondary industry to GDP (gdp2), (2) the ratio of FDI to GDP (fdi), (3) Logarithmic value of urban GDP per capita (lrgdp).

\subsection{Statistical description}

Table 1 Descriptive statistical results of variables

\begin{tabular}{|c|c|c|c|c|c|}
\hline Variable & $\begin{array}{c}\text { Number of } \\
\text { observations }\end{array}$ & Mean & $\begin{array}{c}\text { Standard } \\
\text { Error }\end{array}$ & Minimum & Maximum \\
\hline pm25 & 2,515 & 42.52 & 19.41 & 3.382 & 110.1 \\
\hline asr & 2,515 & 0.307 & 0.461 & 0 & 1 \\
\hline bsr & 2,515 & 0.324 & 0.468 & 0 & 1 \\
\hline axb & 2,515 & 0.949 & 0.220 & 0 & 1 \\
\hline bxb & 2,515 & 0.940 & 0.237 & 0 & 1 \\
\hline abk & 2,515 & 0.130 & 0.337 & 0 & 1 \\
\hline bbk & 2,515 & 0.107 & 0.309 & 0 & 1 \\
\hline ayjs & 2,515 & 0.860 & 0.347 & 0 & 1 \\
\hline byjs & 2,515 & 0.885 & 0.318 & 0 & 1 \\
\hline apg & 2,515 & 0.337 & 0.473 & 0 & 1 \\
\hline bpg & 2,515 & 0.387 & 0.487 & 0 & 1 \\
\hline adx & 2,515 & 0.660 & 0.474 & 0 & 1 \\
\hline bdx & 2,515 & 0.612 & 0.487 & 0 & 1 \\
\hline ajg & 2,515 & 0.669 & 0.471 & 0 & 1 \\
\hline bjg & 2,515 & 0.690 & 0.463 & 0 & 1 \\
\hline ajctb & 2,515 & 0.129 & 0.336 & 0 & 1 \\
\hline bjctb & 2,515 & 0.197 & 0.398 & 0 & 1 \\
\hline axjkp & 2,515 & 0.0934 & 0.291 & 0 & 1 \\
\hline bxjkp & 2,515 & 0.0994 & 0.299 & 0 & 1 \\
\hline azhrz & 2,515 & 0.776 & 0.417 & 0 & 1 \\
\hline bzhrz & 2,515 & 0.705 & 0.456 & 0 & 1 \\
\hline agq & 2,515 & 0.305 & 0.460 & 0 & 1 \\
\hline bgq & 2,515 & 0.316 & 0.465 & 0 & 1 \\
\hline alm & 2,515 & 0.159 & 0.366 & 0 & 1 \\
\hline blm & 2,515 & 0.101 & 0.301 & 0 & 1 \\
\hline anl & 2,515 & 53.13 & 3.529 & 30 & 69 \\
\hline bnl & 2,515 & 50.87 & 3.711 & 35 & 64 \\
\hline axz & 2,515 & 0.0656 & 0.248 & 0 & 1 \\
\hline bxz & 2,515 & 0.0946 & 0.293 & 0 & 1 \\
\hline gdp2 & 2,515 & 48.02 & 10.53 & 14.73 & 89.75 \\
\hline fdi & 2,515 & 0.0176 & 0.0182 & $1.82 e-06$ & 0.198 \\
\hline 1pgdp & 2,515 & 10.60 & 0.706 & 8.555 & 13.19 \\
\hline & & & & & \\
\hline
\end{tabular}

Table 1 reports the descriptive statistical results of the main variables. On the whole, there is a large gap between the environmental governance performance of cities at various levels, the minimum is only 3.382 , and the maximum is as high as 110.1 . By interpreting the characteristics of officials in Table 1, we found that a total of about $30 \%$ of prefecture-level city officials were newly appointed that year; about $95 \%$ of the officials were male officials; about $10 \%$ of officials had undergraduate degrees; about $10 \%$ of officials had graduated $85 \%$; about $35 \%$ of officials who have gone to regular colleges and universities; about $65 \%$ of officials who have gone to party school; about $68 \%$ of those who have studied economics and management majors; about $16 \%$ of those who have been promoted from the basic level; Approximately $10 \% ; 74 \%$ of those who have worked comprehensively; $30 \%$ of those who have worked in state-owned enterprises; about $13 \%$ of those who violated disciplines and corruption; about $8 \%$ of those who have served as principals. The average age of the secretary of the municipal party committee is 53.13, and the average age of the mayor is 50.87 . 


\subsection{Model setting}

This article's measurement model refers to Zang Chuanqin's measurement model [5], using a linear model. The regression model is as follows:

$$
p m 25_{i t}=\alpha_{0}+\alpha_{1} \mathrm{GYTZ}_{\mathrm{it}}+\alpha_{2} \mathrm{X}_{\mathrm{it}}+\varepsilon_{\mathrm{it}}
$$

$p m 25_{i t}$ in the model is an explained variable, which measures the city's environmental governance performance; GYTZ $_{\text {it }}$ is the core explanatory variable of this article, which covers all the above-mentioned variables such as the individual characteristics, education background, and job background of officials; $X_{i t}$ is the control variable of this article Including the ratio of secondary industry to GDP, the ratio of FDI to GDP, and the logarithm of urban GDP per capita; Eit represents a random item. Among them, if the coefficient of the variable $\mathrm{GYTZ}_{\text {it }}$ is significantly positive, it indicates that the official characteristics are not conducive to pollution control and will aggravate the deterioration of the environment. If it is significantly negative, the opposite is true.

\subsection{LASSO regression}

LASSO regression (Least absolute shrinkage and selection operator) was proposed by Robert Tibshirani [7]. It is estimated by the generalized linear model after constructing penalty constraints to impose constraints on the coefficients, to obtain the minimum residual sum of squares that can reduce the variance. Besides, deleting explanatory variables with zero coefficients in the LASSO regression results can effectively solve the problem of multicollinearity between variables, and at the same time realize the problem of variable screening, which plays a role in streamlining the model.

The principle of LASSO regression is to use the minimum sum of squared residuals of OLS as the objective function, and at the same time introduce the L1 norm to the function as a penalty term:

$$
\beta^{\text {lasso }}=\operatorname{argmin}(y-X \beta)^{\prime}(y-X \beta)+\lambda\|\beta\|_{1}
$$

Where $\lambda>0$ is the adjustment parameter to control the penalty intensity and $\|\beta\|_{1}$ is the L1 norm of the parameter vector $\beta$, that is, $\|\beta\|_{1}=\sum_{j=1}^{p}\left|\beta_{\mathrm{j}}\right|$ is the sum of the absolute values of the regression coefficients. In the implementation process, the equation is transformed into:

$$
\begin{aligned}
& \beta^{\text {lasso }}=\operatorname{argmin} \sum_{i=1}^{n}\left(y_{i}-\beta_{0}-\sum_{j=1}^{p} x_{i j} \beta_{j}\right)^{2} \\
& \text { s.t. } \sum_{\mathrm{j}=1}^{p}\left|\beta_{\mathrm{j}}\right| \leq \mathrm{t}
\end{aligned}
$$

In LASSO regression, the default variables obey Gaussian distribution when estimating, and the objective function of the regression is:

$$
\min _{\left(\beta_{0} \beta\right) \in \mathrm{R}^{\mathrm{P}+1}} \frac{1}{2 \mathrm{~N}} \sum_{\mathrm{i}=1}^{n}\left(y_{i}-\beta_{0}-x_{i}^{T} \beta\right)^{2}+\frac{1}{2} \lambda\|\beta\|_{2}^{2}+\alpha\|\beta\|_{1}
$$

when $\alpha=1$, and the parameter $\alpha$ is suitable for situations with high correlation data. Through cross-validation, the value of the adjustment parameter $\lambda$ that minimizes the model estimation error and the estimated coefficient of the corresponding explanatory variable can be obtained, to optimize the model and construct the model with the most explanatory power.

\section{Results \& Discussion}

\section{1 multicollinearity test}

Due to a large number of explanatory variables, the situation of multicollinearity is likely to affect the

\begin{tabular}{|c|c|}
\hline Variable & VIF \\
\hline bpg & 112.8 \\
\hline $\mathrm{bdx}$ & 112.7 \\
\hline azhrz & 64.48 \\
\hline $\mathrm{adx}$ & 62.24 \\
\hline apg & 61.78 \\
\hline bzhrz & 60.67 \\
\hline bjetb & 46.57 \\
\hline ajctb & 42.04 \\
\hline axjkp & 32 \\
\hline bxjkp & 26.34 \\
\hline ayjs & 17.38 \\
\hline abk & 16.79 \\
\hline byjs & 15.38 \\
\hline bbk & 15.17 \\
\hline lpgdp & 1.310 \\
\hline bnl & 1.190 \\
\hline asr & 1.190 \\
\hline gdp2 & 1.190 \\
\hline bsr & 1.180 \\
\hline ajg & 1.170 \\
\hline anl & 1.160 \\
\hline fdi & 1.150 \\
\hline bjg & 1.100 \\
\hline blm & 1.060 \\
\hline bgq & 1.060 \\
\hline bxz & 1.050 \\
\hline axz & 1.050 \\
\hline agq & 1.050 \\
\hline alm & 1.050 \\
\hline$a x b$ & 1.040 \\
\hline $\mathrm{bxb}$ & 1.040 \\
\hline
\end{tabular}
estimation results of OLS, so the multicollinearity test is first performed. Table 2 reports the multicollinearity test results.

Table 2 Results of multicollinearity test

Generally speaking, if vif is greater than 10 , it means that the variable may have obvious multicollinearity [8]. From Table 2, it can be seen that the explanatory variables used in this article have a severe multicollinearity problem. According to Table 2, there are 14 variables with vif values greater than 10 , and the largest one even reaches 112.8 . It can be considered that there is serious multicollinearity among the explanatory variables. Therefore, it is necessary to choose a regression method that can overcome multicollinearityLASSO regression.

Among them, $0 \leq \alpha \leq 1$, LASSO estimation is performed 


\subsection{LASSO regression process and results}

For the LASSO series of penalty estimators, it is necessary to determine the optimal adjustment parameter, such as $\lambda$ or $\alpha$. This article uses the 10 -fold cross-validation method provided by Stata16 to select the optimal adjustment parameter $\lambda$. Figure 1 shows the coefficients of the data in the LASSO regression process. Table 3 shows the important explanatory variables and coefficients selected by LASSO regression in the graph of changes with parameters.

It can be seen from the Figure 1 that when the adjustment parameter $\lambda=0$, the result of LASSO regression is the result of OLS regression, but when $\lambda$ is large enough, the penalty is too large, resulting in all regression coefficients being 0 .

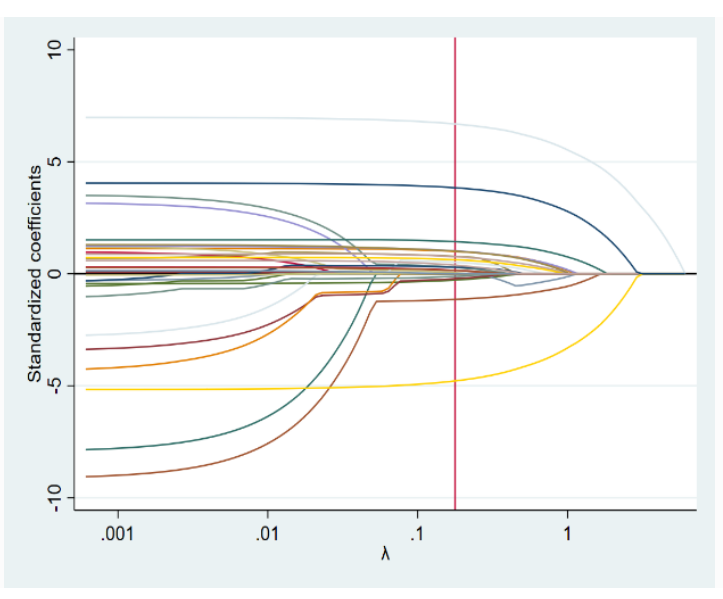

Figure 1 Path coefficient diagram

Table 3 LASSO regression results

\begin{tabular}{|c|c|}
\hline Variable & Coefficient \\
\hline bxz & 1.434 \\
\hline apg & -1.143 \\
\hline bgq & 1.022 \\
\hline anl & 1.013 \\
\hline bxb & 0.998 \\
\hline bdx & 0.778 \\
\hline ajg & 0.763 \\
\hline bjg & 0.627 \\
\hline ajctb & 0.514 \\
\hline bnl & 0.384 \\
\hline bjctb & 0.294 \\
\hline abk & 0.285 \\
\hline axb & -0.279 \\
\hline axjkp & -0.217 \\
\hline agq & 0.154 \\
\hline blm & 0.148 \\
\hline bzhrz & -0.116 \\
\hline byjs & 0.0472 \\
\hline
\end{tabular}

The coefficients of the core explanatory variables selected by LASSO regression shown in Table 3 are the coefficients after data standardization. All explanatory variables must be standardized before LASSO regression because the unit or value range of the variables is The results of penalty regression, such as LASSO, has a substantial impact. Standardization is to subtract the sample mean of the variable and divide by its standard deviation, so that the mean of each explanatory variable is
0 and the standard deviation is 1 , thereby eliminating the unit and value of the variable. The influence of range on LASSO regression.

Through LASSO regression filtered variables and their coefficients, we found that:

(1) The secretary of the municipal party committee who has attended a common university is beneficial to the pollution control of the city. The reason may be that, compared with the municipal party committee secretary who has not attended a normal university, the municipal party committee secretary who has attended a normal university has stronger learning ability, masters more scientific and cultural knowledge, and can better handle the relationship between economic development and environmental protection. While developing the economy, we can also handle environmental issues well.

(2) The municipal party committee secretary and a mayor who has worked in state-owned enterprises are not conducive to the pollution control of the city. Among them, the mayor who has worked in a state-owned enterprise is less conducive to the pollution control of the city than the secretary of the municipal party committee. Although state-owned enterprises have government participation, the development of state-owned enterprises covers a narrower area than the development of cities. State-owned enterprises focus on corporate interests, that is, profitability. The municipal party secretary and a mayor who has worked in state-owned enterprises may be affected by this job. Compared with those who have not worked in a state-owned enterprise, they value the development of the urban economy more and ignore the pollution control of the city;

(3) The increasing age of the municipal party committee secretary and the mayor is not conducive to urban pollution control. The increase in the age of the secretary of the municipal party committee has a greater impact than the increase in the age of the mayor. This may be because older officials have less energy and cannot handle all aspects of political affairs. Compared with younger officials, they cannot coordinate the relationship between economic development and pollution control. At the same time, the older officials will have lower their desire for political achievements. Some even older officials will relax and even allow some polluting enterprises to pursue a more comfortable life for their benefit and want to live a more comfortable life after retirement. Self-interest.

(4) The municipal party committee secretary and a mayor who has studied economics and management are not conducive to urban pollution control. The reason may be that people who have studied economics and management are generally affected by the assumption of "rational people", they are more cautious and rational in handling things, and they are inclined and able to achieve "profit maximization". Such officials are generally good at economic development, but in order to achieve "profit maximization", it is easy to cause environmental damage. Therefore, officials who have studied economics and management are not conducive to urban pollution control.

(5) The municipal party committee secretary and mayor promoted from the grassroots are not conducive to 
urban pollution control. The officials promoted at the grassroots level used to serve as officials of units below the prefecture-level city, such as the county-level township level, and are now promoted to the municipal party secretary and mayor of prefecture-level cities. This transformation involves a considerable change in the scope and content of management. However, officials promoted at the grassroots level may not be able to deal with pollution control problems in cities due to insufficient knowledge and control of environmental protection policies.

(6) The secretary of the municipal party committee sent from the sky is conducive to the pollution control of the city. The possible reason is that such officials have served in units above the prefecture-level and city-level, so they have a better overall view and can coordinate the relationship between economic development and environmental protection. At the same time, officials sent from the sky may have a greater desire for political performance for "exercising". Compared with other officials, they pay more attention to environmental issues while developing the economy, so they have a positive effect on urban pollution control.

(7) A mayor who holds a comprehensive post is conducive to urban pollution control. Perhaps the mayor with this kind of experience has served in units below, and above the prefecture-level city level, so he has a wealth of governance experience and can better coordinate the city's environmental and economic development issues, thereby conducive to pollution control.

(8) The secretary of the municipal party committee whose final degree is a bachelor's degree and the mayor whose final degree is a graduate degree are not conducive to the city's environmental governance. Because economic development and pollution control are difficult to take into account at the same time, it requires a strong personal ability to develop the economy and the environment at the same time. Research shows that officials with undergraduate and graduate degrees positively impact the development of the regional economy. It shows that such officials may be more focused on economic development between economic development and environmental governance, and it is challenging to take environmental governance into account, resulting in a decline in environmental governance performance.

(9) The mayor who has committed corruption and violated discipline is not conducive to urban pollution control. The possible reason is that officials who have been corrupted and violated disciplines have benefited from some companies so that the officials have relaxed or even allowed some corporate actions that pollute the environment.

(10) According to the results of the LASSO regression, it is also found that for mayors who have attended a party school, it is not conducive to urban pollution control.

Although LASSO regression screened out the variables of the mayor who served as the principal, the gender of the municipal party committee secretary, and the gender of the mayor, because the sample size of these characteristics is small (less than $10 \%$ ), the reference value is not great.

\section{Conclusions}

Based on the panel data of 285 prefecture-level cities across the country from 2010 to 2018 , this paper uses the LASSO regression model to empirically analyze the impact of local officials' characteristics on environmental governance performance. The study found that multiple characteristics of local officials will have an impact on the environmental governance performance within their jurisdiction. Among them, the secretary of the municipal party committee who has served in ordinary colleges and universities, the secretary of the municipal party committee, and the mayor who served comprehensively positively affect environmental governance in the jurisdiction. The increase in the age of the municipal party secretary and mayor, municipal party secretary and a mayor who has worked in state-owned enterprises, the municipal party secretary and a mayor who have studied economics and management, the municipal party secretary and mayor promoted from the grassroots, and the final education The secretary of the municipal party committee who is an undergraduate, the mayor whose final degree is a postgraduate, the mayor who has committed corruption and violated discipline, and the mayor who has been to a party school are not conducive to environmental governance in the jurisdiction.

Environmental issues have gradually become an essential factor restricting China's sustainable development and economic stability. The government plays a vital role in the governance of environmental issues. Local governments, in particular, are duty-bound to solve environmental governance issues. Local government officials are directly related to the government's decision-making, so how to balance economic development with the relationship between environmental governance tests the level of officials. This article's research results help to understand the role of individual differences in local officials in environmental governance and can also provide reference suggestions for cadres and personnel reform.

\section{Acknowledgments}

This paper is supported by National College Student Innovation and Entrepreneurship Training Program (202010404018).

\section{References}

1. Liu Xiaofeng. An Empirical Analysis of the Impact of Social Capital on China's Environmental Governance Performance $[\mathrm{J}]$. China Population, Resources and Environment, 2011, 21(3): 20-24.

2. Yu Guansheng. Trade Openness, FDI and Environmental Pollution Control-Taking Industrial Wastewater Control as an Example [J]. China Economic Issues, 2011 (6): 83-89.

3. Zhang Yabin, Ma Chen, Jin Peizhen. Evaluation of my country's environmental governance investment performance and its influencing factors-_-SBM- 
TOBIT two-stage model based on panel data $[\mathrm{J}]$. Economic Management, 2014, 36(4): 170-179.

4. Fu Caifang. Economic analysis of environmental governance performance of local governments: empirical evidence from 196 prefecture-level cities in China [J]. Technoeconomics, 2020, 39(07): 193-199.

5. Zang Chuanqin, Chu Shuai. Characteristics of Local Officials, Official Communication and Environmental Governance_ _ Based on the Empirical Evidence of 25 Provincial Units in China from 2003 to 2013[J]. Collected Economics and Finance, 2016(11): 105-112.

6. Cai Fang, Du Yang, Wang Meiyan. The transformation of economic development mode and the internal driving force of energy saving and emission reduction [J] . Economic Research, 2008(6): 4-11, 36.

7. Robert Tibshirani. Regression Shrinkage and Selection Via the LASSO. 1996, 58(1):267-288.

8. Zhou Yanfang, Wen Youdong, Yang Jun. Research on the Influencing Factors of the Future Price Expectation Index of Urban Residents[J]. Statistics and Decision, 2020, 36(04): 40-44. 